\title{
MATCHINGS AND THE VARIANCE OF LIPSCHITZ FUNCTIONS*
}

\author{
Franck Barthe $^{1}$ and NeIl O'Connell ${ }^{2}$
}

\begin{abstract}
We are interested in the rate function of the moderate deviation principle for the twosample matching problem. This is related to the determination of 1-Lipschitz functions with maximal variance. We give an exact solution for random variables which have normal law, or are uniformly distributed on the Euclidean ball.
\end{abstract}

Mathematics Subject Classification. 60D05, 60F10, 26D10.

Received September 24, 2007.

\section{INTRODUCTION}

Let $X_{i}$ and $Y_{i}$ be independent random variables in $\mathbb{R}^{d}$ with common law $\mu$, and set

$$
T_{n}=\inf _{\sigma \in S_{n}} \sum_{i=1}^{n}\left|X_{i}-Y_{\sigma(i)}\right|
$$

where $S_{n}$ denotes the set of permutations of $\{1, \ldots, n\}$. In the case where $d=2$ and $\mu$ is the uniform distribution on the unit square, this is the canonical "two-sample matching problem". In this case, Ajtai et al. [1] prove the following: there exists $K>0$ such that

$$
\frac{1}{K}(n \log n)^{1 / 2}<T_{n}^{1}<K(n \log n)^{1 / 2},
$$

with probability $1-o(1)$. Refinements of this result, as well as concentration inequalities, were obtained by Shor [10] and Talagrand [11]. It is still an open problem to determine if $(n \log n)^{-1 / 2} T_{n}^{1}$ actually converges, even in expectation. In [4], large and moderate deviations results were obtained in a fairly general setting by exploiting a connection with the Monge-Kantorovich problem. Let $E$ be a Borel subset of $\mathbb{R}^{d}$ and assume that $\mu \in M_{1}(E)$, the space of probability measures on $E$. The Monge-Kantorovich distance on $M_{1}(E)$ is defined by

$$
W_{1}(\mu, \nu)=\inf \left\{\int_{E \times E}|x-y| \pi(\mathrm{d} x, \mathrm{~d} y): \pi \in \Pi(\mu, \nu)\right\},
$$

Keywords and phrases. Matching problem, large deviations, variance, spectral gap, Euclidean ball.

* Research supported by Science Foundation Ireland Grant Number SFI 04-RP1-I512.

1 Institut de Mathématiques de Toulouse, CNRS UMR 5219, Université Paul Sabatier, 31062 Toulouse Cedex 09, France; barthe@math.univ-toulouse.fr

2 Mathematics Institute, University of Warwick, Coventry CV4 7AL, UK. 
where $\Pi(\mu, \nu)$ is the space of all Borel probability measures $\pi$ on $\mathbb{R}^{d} \times \mathbb{R}^{d}$ with fixed marginals $\mu(\cdot)=\pi\left(\cdot \times \mathbb{R}^{d}\right)$ and $\nu=\pi(E \times \cdot)$. We recall that, if $\mathcal{F}$ denotes the set of Lipschitz functions on $E$ with Lipschitz constant 1 , then

where

$$
W_{1}(\mu, \nu)=\|\mu-\nu\|_{\mathcal{F}}
$$

$$
\|\nu\|_{\mathcal{F}}=\sup \left\{\left|\int f \mathrm{~d} \nu\right|: f \in \mathcal{F}\right\}
$$

If $E$ is compact, then $W_{1}$ metrises the weak topology on $M_{1}(E)$. Define the empirical measures

$$
L_{n}=\frac{1}{n} \sum_{i=1}^{n} \delta_{X_{i}}, \quad M_{n}=\frac{1}{n} \sum_{i=1}^{n} \delta_{Y_{i}} .
$$

It is an immediate consequence of the Birkhoff-von Neumann theorem that

$$
\frac{1}{n} T_{n}=W_{1}\left(L_{n}, M_{n}\right)
$$

Starting with this identity, large and moderate deviations results for the matching problem were obtained in [4]. Before stating these, we recall some definitions from large deviation theory.

Let $Z_{n}$ be a sequence of random variables defined on a probability space $(\Omega, \mathcal{F}, \mathbb{P})$, with values in a topological space $\mathcal{X}$ equipped with the Borel $\sigma$-algebra $\mathcal{B}$. We say that the sequence $Z_{n}$ satisfies the large deviation principle (LDP) with rate function $I$ and speed $n$, if for all $B \in \mathcal{B}$,

$$
-\inf _{x \in B^{\circ}} I(x) \leq \liminf _{n} \frac{1}{n} \log \mathbb{P}\left(Z_{n} \in B\right) \leq \limsup _{n} \frac{1}{n} \log \mathbb{P}\left(Z_{n} \in B\right) \leq-\inf _{x \in \bar{B}} I(x) .
$$

Let $\left(a_{n}\right)_{n \geq 1}$ be an increasing, positive sequence such that

$$
a_{n} \rightarrow \infty, \quad \frac{a_{n}}{\sqrt{n}} \rightarrow 0 .
$$

We say the the sequence $Z_{n}$ satisfies the moderate deviation principle (MDP) with rate function $J$ and speed $a_{n}^{-2}$, if for all $B \in \mathcal{B}$,

$$
\begin{aligned}
-\inf _{x \in B^{\circ}} J(x) & \leq \liminf _{n} \frac{1}{a_{n}^{2}} \log \mathbb{P}\left(\frac{\sqrt{n}}{a_{n}} Z_{n} \in B\right) \\
& \leq \limsup _{n} \frac{1}{a_{n}^{2}} \log \mathbb{P}\left(\frac{\sqrt{n}}{a_{n}} Z_{n} \in B\right) \leq-\inf _{x \in \bar{B}} J(x) .
\end{aligned}
$$

Denote by $\mathcal{F}_{0}$ the set of $f \in \mathcal{F}$ such that $\int f d \mu=0$. For $\theta \in \mathbb{R}$, define

$$
\Lambda_{f}(\theta)=\log \mathbf{E}_{\mu}\left[\mathrm{e}^{\theta f}\right]=\log \int_{E} \mathrm{e}^{\theta f(x)} \mathrm{d} \mu(x), \quad L_{f}(\theta)=\Lambda_{f}(\theta)+\Lambda_{f}(-\theta),
$$

and for $x \in \mathbb{R}$, set

$$
L_{f}^{*}(x)=\sup _{\theta \in \mathbb{R}} \theta x-L_{f}(\theta) .
$$

The following theorem is a slight generalisation of the main result in [4], where it was assumed that $E$ is compact for the LDP and that $E$ is the unit square for the MDP. The proof is essentially the same, combining large and moderate deviation results for empirical measures given in $\mathrm{Wu}$ [13] (see Sect. 3 for the unbounded case, which relies heavily on earlier work of Ledoux [5]) with convergence rates for empirical measures in the 
Monge-Kantorovich distance due to Dudley [3] and, for the unbounded case, Kalashnikov and Rachev (see [9], Th. 11.1.6).

Theorem 1. Assume that either $E$ is compact or $\mu$ is the standard Gaussian measure on $\mathbb{R}^{d}$.

(i) The sequence $T_{n} / n$ satisfies the large deviation principle in $\mathbb{R}_{+}$with rate function

$$
I(x)=\inf _{f \in \mathcal{F}_{0}} L_{f}^{*}(x) .
$$

(ii) Let $a_{n}$ be a positive sequence satisfying the following conditions:

(1) as $n \rightarrow \infty, \frac{a_{n}}{\sqrt{n}} n^{1 / k} \rightarrow \infty$, for some $k>\max \{d, 2\}$;

(2) for some $\delta, A>0, a_{n m} \leq A m^{-\delta+1 / 2} a_{n}$, for all $n, m>0$.

Then the sequence $T_{n} / \sqrt{n} a_{n}$ satisfies the $M D P$ in $\mathbb{R}_{+}$with speed $a_{n}^{-2}$ and rate function

$$
J(x)=\frac{x^{2}}{4 \sup _{f \in \mathcal{F}_{0}} \int_{E} f^{2} d \mu} .
$$

In the case where $\mu$ is uniform measure on the unit square, it was conjectured in [4] that the infimum in (1.6) and the supremum in (1.7) are both achieved by $f=\varphi$, where

$$
\varphi(x, y)=\frac{x+y}{\sqrt{2}}, \quad-\frac{1}{2} \leq x, y \leq \frac{1}{2}
$$

which yields $J(x)=3 x^{2}$ and $I=L_{\varphi}^{*}$, where

$$
L_{\varphi}(\theta)=2 \log \left[\frac{4}{\theta^{2}}\left(\cosh \left(\frac{\theta}{4 \sqrt{2}}\right)-1\right)\right] .
$$

Note that, in this case, any affine function $f \in \mathcal{F}_{0}$ with $|\nabla f|=1$ has the same variance. As explained in [4], the conjecture is true if, for all $\theta \in \mathbb{R}$, the supremum of $L_{f}(\theta)$ over $f \in \mathcal{F}_{0}$ is achieved by $f=\varphi$. Note that this is true if, for all positive, even integers $r$, the supremum of the $r^{t h}$ cumulant $\Lambda_{f}^{(r)}(0)$ over $f \in \mathcal{F}_{0}$ is achieved by $f=\varphi$. In this paper, we compute the rate functions $I$ and $J$ explicitly in the case where $\mu$ is the standard Gaussian measure on $\mathbb{R}^{d}$. In this case, the variance and all exponential moments are maximised by the linear function $\varphi(x)=x_{1}$. We also compute the moderate deviations rate function $J$ in the case where $\mu$ is the uniform measure on the unit ball in $\mathbb{R}^{d}$; in this case too the variance is maximised by $\varphi(x)=x_{1}$. Throughout the paper, the space $\mathbb{R}^{d}$ is equipped with the canonical Euclidean structure, with norm $|\cdot|$ and scalar product $x \cdot y$.

\section{Gaussian samples}

In this section we show that for standard Gaussian samples the rate functions in the large and moderate deviation principles can be explicitly calculated. Let $\gamma^{d}$ denote the standard Gaussian measure on $\mathbb{R}^{d}$.

Proposition 2. For $E=\left(\mathbb{R}^{d},|\cdot|, \gamma^{d}\right)$,

$$
I(x)=J(x)=\frac{x^{2}}{4}
$$

Proof. For the moderate deviation problem, we use the classical spectral gap inequality for the Gaussian measure (see e.g. [6]): for every locally Lipschitz $f: \mathbb{R}^{d} \rightarrow \mathbb{R}$

$$
\operatorname{Var}_{\gamma^{d}}(f) \leq \int|\nabla f|^{2} \mathrm{~d} \gamma^{d}
$$


This is an equality for affine functions. If $f$ is 1 -Lipschitz, this yields $\operatorname{Var}_{\gamma^{d}}(f) \leq 1$, and this bound is best possible (e.g. for $f(x)=x_{1}$ ). Hence $\sup _{\mathcal{F}_{0}} \int f^{2} \mathrm{~d} \gamma^{d}=1$ and this allows to calculate $J$.

For the large deviation problem, our starting point is Talagrand's transportation cost inequality [12]. Denot$\operatorname{ing} W_{p}(\mu, \nu):=\inf \left\{\int|x-y|^{p} \mathrm{~d} \pi(x, y)\right\}^{1 / p}$ where the infimum is with respect to probability measures on $E \times E$ with marginals $\mu$ and $\nu$, this inequality reads as: for all probability measure $f \mathrm{~d} \gamma^{d}$ on $\mathbb{R}^{d}$,

$$
W_{2}\left(\gamma^{d}, f \mathrm{~d} \gamma^{d}\right) \leq \sqrt{2 \operatorname{Ent}_{\gamma^{d}}(f)} .
$$

This inequality is an equality when $f(x)=\exp \left(\langle t, x\rangle-|t|^{2} / 2\right)$, in which case the optimal transport is just a translation by $t$. Consequently by Cauchy-Schwarz inequality

$$
W_{1}\left(\gamma^{d}, f \mathrm{~d} \gamma^{d}\right) \leq \sqrt{2 \operatorname{Ent}_{\gamma^{d}}(f)}
$$

and the inequality is still sharp for the above examples (the reason being that the translation is the optimal transportation for $W_{1}$ and $W_{2}$; in this case the length of the displacement is constant so there was no loss when we applied Cauchy-Schwarz). So this inequality can be rewritten as for $a \geq 0$

$$
\inf \left\{\operatorname{Ent}_{\gamma^{d}}(f) ; W_{1}\left(\gamma^{d}, f \mathrm{~d} \gamma^{d}\right)=a\right\}=\frac{a^{2}}{2} .
$$

There is also a dual reformulation on the transportation cost inequality, put forward by Bobkov and Götze [2]: for all Lipschitz functions $f: \mathbb{R}^{d} \rightarrow \mathbb{R}$, and for all $t \in \mathbb{R}$

$$
\int \mathrm{e}^{t\left(f-\int f \mathrm{~d} \gamma^{d}\right)} \mathrm{d} \gamma^{d} \leq \mathrm{e}^{\frac{t^{2}}{2}}
$$

The above inequality becomes an equality when $f(x)=x_{1}$ for example. It follows that for $f \in \mathcal{F}_{0}$,

$$
L_{f}(\theta)=\log \left(\int \mathrm{e}^{\theta f} \mathrm{~d} \gamma^{d}\right)+\log \left(\int \mathrm{e}^{-\theta f} \mathrm{~d} \gamma^{d}\right) \leq \theta^{2}=L_{\varphi}(\theta)
$$

where we have set $\varphi(x)=x_{1}$. As explained in Section 4 of [4] this implies that for $x \geq 0, I(x)=L_{\varphi}^{*}(x)=\frac{x^{2}}{4}$.

\section{EuClideAn Unit BALL}

The goal of this section is to calculate the rate function in the moderate deviation principle for random variables with uniform distribution on the $d$-dimensional Euclidean ball $B_{2}^{d} \subset \mathbb{R}^{d}$ :

Theorem 3. For $E=\left(B_{2}^{d},|\cdot|, \mathbf{1}_{B_{2}^{d}}(x) \frac{\mathrm{d} x}{\operatorname{Vol}\left(B_{2}^{d}\right)}\right)$,

$$
J(x)=\frac{d+2}{4} x^{2} .
$$

In view of (1.7), this is a consequence of the following result, which asserts that among 1-Lipschitz functions, linear functions have maximal variance on the ball:

Theorem 4. Let $f: B_{2}^{d} \rightarrow \mathbb{R}$ be a 1-Lipschitz function (for the Euclidean distance) such that $\int_{B_{2}^{d}} f(x) \mathrm{d} x=0$. Then

$$
\int_{B_{2}^{d}} f^{2}(x) \mathrm{d} x \leq \int_{B_{2}^{d}} x_{1}^{2} \mathrm{~d} x=\frac{\operatorname{Vol}\left(B_{2}^{d}\right)}{d+2} .
$$


Proof. The direct approach to such a statement consists in proving that there exist maximizing functions, by Ascoli theorem. Then perturbing such a maximizer $f$, one is easily convinced that it should satisfy the Eikonal equation $|\nabla f|=1$ a.e. in $B_{2}^{d}$. However we do not know how to push this method further. This is why we adopt another strategy, which is unfortunately very specific to the ball. We are going to derive, by classical spectral analysis, a Poincaré type inequality of the following form: for every locally Lipschitz $f: B_{2}^{d} \rightarrow \mathbb{R}$ with $\int_{B_{2}^{d}} f=0$,

$$
\int_{B_{2}^{d}} f(x)^{2} \mathrm{~d} x \leq \int_{B_{2}^{d}} \varphi(x)|\nabla f(x)|^{2} \mathrm{~d} x,
$$

where the function $\varphi$ is adjusted so that the inequality becomes and equality for linear functionals $f_{0}(x)=x \cdot e$ for any $e \in \mathbb{R}^{d}$. As a consequence if $\int_{B_{2}^{d}} f=0$,

$$
\int_{B_{2}^{d}} f^{2} \leq \int_{B_{2}^{d}} \varphi|\nabla f|^{2} \leq\left(\int_{B_{2}^{d}} \varphi\right)\left(\sup _{B_{2}^{d}}|\nabla f|\right)^{2} .
$$

Note that both inequalities become equalities for the function $f(x)=x_{1}$ (and more generally for $f(x)=x \cdot e$ for any unit vector $e \in \mathbb{R}^{d}$ ). Hence the inequality between the first and the last term can be written as

$$
\int_{B_{2}^{d}} f^{2} \leq\left(\int_{B_{2}^{d}} x_{1}^{2} \mathrm{~d} x\right)\left(\sup _{B_{2}^{d}}|\nabla f|\right)^{2} .
$$

For 1-Lipschitz functions we would get as claimed $\int_{B_{2}^{d}} f^{2} \leq \int_{B_{2}^{d}} x_{1}^{2} \mathrm{~d} x$.

Let us briefly sketch how to choose $\varphi$. Assume that for all $f: B_{2}^{d} \rightarrow \mathbb{R}$ with $\int_{B_{2}^{d}} f=0$,

$$
\int_{B_{2}^{d}} f(x)^{2} \mathrm{~d} x \leq \int_{B_{2}^{d}} \varphi(x)|\nabla f(x)|^{2} \mathrm{~d} x
$$

and that there is equality for a non-constant function $f_{0}$. We follow the classical method to obtain the corresponding Euler-Lagrange equation: let $u$ be a smooth bounded function with $\int_{B_{2}^{d}} u=0$. Then $\int\left(f_{0}+\varepsilon u\right)^{2} \leq$ $\int \varphi\left|\nabla\left(f_{0}+\varepsilon u\right)\right|^{2}$ for every $\varepsilon \in \mathbb{R}$. Since there is equality for $\varepsilon=0$, the first order term in $\varepsilon$ vanishes, that is $\int f_{0} u=\int \varphi \nabla f_{0} . \nabla u$. Stokes formula then yields

$$
\int_{B_{2}^{d}} f_{0} u=-\int_{B_{2}^{d}} u \nabla \cdot\left(\varphi \nabla f_{0}\right)+\int_{S^{d-1}} u \varphi n \cdot \nabla f_{0},
$$

where $n$ denotes the unit outer normal to the sphere. Since this is true for every $u$ with integral 0 (i.e. orthogonal to constants in $\left.L^{2}\left(B_{2}^{d}, \mathrm{~d} x\right)\right)$ we obtain there is a constant $c$ such that $f_{0}$ satisfies

$$
\begin{cases}f_{0}+\nabla \cdot\left(\varphi \nabla f_{0}\right)=c & \text { on } B_{2}^{d} \\ \varphi n \cdot \nabla f_{0}=0 & \text { on } S^{d-1}\end{cases}
$$

If $e$ is a unit vector in $\mathbb{R}^{d}$, and $f_{0}(x)=x \cdot e$ is an equality case of a Poincaré inequality involving a smooth function $\varphi$ then necessarily $\varphi$ is identically zero on the unit sphere $S^{d-1}$ and there exists a constant $c_{e}$ such that

$$
x \cdot e+\nabla \varphi(x) \cdot e=c_{e}, \quad \forall x \in B_{2}^{d} .
$$

This is assumed to be true for every unit vector $e$ so that $x+\nabla \varphi(x)$ is actually independent of $x \in B_{2}^{d}$. Hence here exist $a \in \mathbb{R}, b \in \mathbb{R}^{d}$ such that $\varphi(x)=a-b \cdot x-|x|^{2} / 2$. Since $\varphi$ vanishes on the unit sphere we conclude

$$
\varphi(x)=\frac{1-|x|^{2}}{2}, \quad x \in B_{2}^{d} .
$$


The Poincaré inequality involving this function is proved in the next theorem.

Theorem 5. Let $d \geq 1$ and integer. Let $f: B_{2}^{d} \rightarrow \mathbb{R}$ be a Lipschitz function such that, $\int_{B_{2}^{d}} f=0$. Then

$$
\int_{B_{2}^{d}} f^{2}(x) \mathrm{d} x \leq \int_{B_{2}^{d}} \frac{1-|x|^{2}}{2}|\nabla f|^{2} \mathrm{~d} x
$$

with equality when $f$ is a linear function.

Proof. For $d=1$ this may be proved using the classical method:

$$
\begin{aligned}
\int_{-1}^{1} f^{2} & =\frac{1}{4} \int_{[-1,1]^{2}}(f(y)-f(x))^{2} \mathrm{~d} x \mathrm{~d} y=\frac{1}{2} \int_{-1<x<y<1}(f(y)-f(x))^{2} \mathrm{~d} x \mathrm{~d} y \\
& =\frac{1}{2} \int_{-1<x<y<1}\left(\int_{x}^{y} f^{\prime}(t) \mathrm{d} t\right)^{2} \mathrm{~d} x \mathrm{~d} y \leq \frac{1}{2} \int_{-1<x<y<1}(y-x)\left(\int_{x}^{y} f^{\prime}(t)^{2} \mathrm{~d} t\right) \mathrm{d} x \mathrm{~d} y \\
& =\int_{-1}^{1} \frac{1-t^{2}}{2} f^{\prime}(t)^{2} \mathrm{~d} t .
\end{aligned}
$$

For $d \geq 2$ the statement follows from the next theorem and an approximation argument, based on the density of polynomials in the space $H^{1}\left(B_{2}^{d}\right)$.

Theorem 6. Let $d \geq 2$. Let $f$ be a polynomial function in $d$ variables, such that $\int_{B_{2}^{d}} f=0$. Then

$$
\int_{B_{2}^{d}} f^{2}(x) \mathrm{d} x \leq \int_{B_{2}^{d}} \frac{1-|x|^{2}}{2}|\nabla f|^{2} \mathrm{~d} x
$$

with equality if and only if $f$ is a linear function.

Proof. It is enough to prove that for every integer $m$ the inequality is valid for $f \in \mathbb{R}_{m}\left[T_{1}, \ldots, T_{d}\right]$, the set of polynomials in $d$ variables with total degree at most $m$. Let us consider the operator

$$
L:=\nabla \cdot\left(\frac{1-|x|^{2}}{2} \nabla f\right)=\frac{1-|x|^{2}}{2} \Delta f-x \cdot \nabla f .
$$

Note that when $f \in \mathbb{R}_{m}\left[T_{1}, \ldots, T_{d}\right]$ is a polynomial of total degree at most $m$ then so is $L f$. Moreover for smooth functions $f, g$ on $\mathbb{R}^{d}$ it holds

$$
\begin{aligned}
\int_{B_{2}^{d}} \frac{1-|x|^{2}}{2} \nabla f \cdot \nabla g \mathrm{~d} x & =-\int_{B_{2}^{d}} f \nabla \cdot\left(\frac{1-|x|^{2}}{2} \nabla g\right) \mathrm{d} x+\int_{S^{n-1}} \frac{1-|x|^{2}}{2} f(x) x \cdot \nabla g(x) \mathrm{d} x \\
& =-\int_{B_{2}^{d}} f L g .
\end{aligned}
$$

In particular $\int_{B_{2}^{d}} f L g=\int_{B_{2}^{d}} L f g$, so $L$ can be viewed as a self-adjoint operator of $\mathbb{R}_{m}\left[T_{1}, \ldots, T_{d}\right]$ equipped with the scalar product $(f, g) \mapsto \int_{B_{2}^{d}} f g$. It follows that the restriction of $L$ to $\mathbb{R}_{m}\left[T_{1}, \ldots, T_{d}\right]$ can be diagonalized. The relation

$$
\int_{B_{2}^{d}} \frac{1-|x|^{2}}{2}|\nabla f|^{2} \mathrm{~d} x=-\int_{B_{2}^{d}} f L f
$$

also implies that the eigenvalues of $f$ are non-positive and that the kernel of $L$ is the set of constant functions.

We will be done if we prove that the none of the eigenvalues of $L$ (on any $\mathbb{R}_{m}\left[T_{1}, \ldots, T_{d}\right]$ ) is in $(-1,0)$, i.e. that $L$ has spectral gap 1 . Indeed if $0 \geq-\lambda_{1} \geq \cdots \geq \lambda_{K}$ denote the non-zero eigenvalues of $L$ (with repetition) and 
$\left(f_{0}=\operatorname{Vol}\left(B_{2}^{d}\right)^{-1 / 2}, f_{1}, \ldots, f_{K}\right)$ is an orthonormal basis of corresponding eigenvectors, the hypothesis $\int_{B_{2}^{d}} f=0$ means that $f$ is orthogonal to constant functions (i.e. the kernel of $L$ ). Hence one can write

$$
f=\sum_{i \geq 1} c_{i} f_{i} \quad \text { and } \quad L f=-\sum_{i \geq 1} c_{i} \lambda_{i} f_{i} .
$$

Consequently

$$
\int_{B_{2}^{d}} f^{2}=\sum_{i \geq 1} c_{i}^{2} \text { and } \int_{B_{2}^{d}} \frac{1-|x|^{2}}{2}|\nabla f|^{2} \mathrm{~d} x=\int_{B_{2}^{d}} f(-L f)=\sum_{i \geq 1} \lambda_{i} c_{i}^{2} .
$$

So the claimed inequality follows from $\lambda_{i} \geq 1$ for all non-zero (opposite) eigenvalues.

Let $-\lambda$ be a non-zero eigenvalue of $L$ on $\mathbb{R}_{m}\left[T_{1}, \ldots, T_{d}\right]$ and let $f$ be a corresponding eigenfunction. Plainly $f$ is not constant and verifies $L f=-\lambda f$. Let $k \geq 1$ denote the total degree of $f$. Let us write $f=g+h$ where $h$ has total degree at most $k-1$ and $g$ is a homogeneous polynomial of degree $k$ and identify the terms of degree $k$ in the latter equality. Note that

$$
x \cdot \nabla\left(x_{1}^{a_{1}} \cdots x_{n}^{a_{n}}\right)=\left(a_{1}+\cdots+a_{n}\right) x_{1}^{a_{1}} \cdots x_{n}^{a_{n}} \quad \text { and } \quad \Delta\left(x_{1}^{a_{1}} \cdots x_{n}^{a_{n}}\right)=\sum_{i=1}^{n} a_{i}\left(a_{i}-1\right) x_{1}^{a_{1}} \cdots x_{i}^{a_{i}-2} \cdots x_{n}^{a_{n}} .
$$

So in the particular case $k=1$, it holds $-\lambda f=-L f=-L g=-x . \nabla g=-g$ so $\lambda=1$ so $f=g, h=0$ and $\lambda=1$. Hence among functions of degree 1, linear functions are the only eigenfunctions; the corresponding eigenvalue is -1 . This is not a surprise in view of our choice of $\varphi$.

Assuming now that $k \geq 2$, we see that

$$
L f=L g+L h=\left(-\frac{|x|^{2}}{2} \Delta g-x \cdot \nabla g\right)+\left(L h+\frac{1}{2} \Delta g\right)
$$

where the first term is homogeneous of degree $k$ and the second one is of degree at most $k-1$. Identifying terms of degree $k$ in the equation $L f=-\lambda f$ thus yields

$$
\frac{|x|^{2}}{2} \Delta g+x \cdot \nabla g=\lambda g, \quad x \in B_{2}^{d} .
$$

Since $g$ is homogeneous of degree $k$ the above equation will allow us to get information about $\lambda$. First recall that $x \cdot \nabla g=k g$. Also if we consider the 0-homogeneous function $\tilde{g}(x)=g(x /|x|)$, then for $x \neq 0, g(x)=|x|^{k} \tilde{g}(x)$. Differentiating twice we get for $x \neq 0$

$$
\begin{aligned}
\Delta g(x) & =\Delta\left(|x|^{k}\right) \tilde{g}(x)+\nabla\left(|x|^{k}\right) \cdot \nabla \tilde{g}(x)+|x|^{k} \Delta \tilde{g}(x) \\
& =k(k+d-2)|x|^{k-2} \tilde{g}(x)+|x|^{k} \Delta \tilde{g}(x),
\end{aligned}
$$

since the gradient of $\tilde{g}$ is tangential and the one of $|x|^{k}$ is radial. Recall that the restriction of $\Delta \tilde{g}$ to the unit sphere $S^{d-1}$ coincides with the spherical gradient of $g$, denoted by $\Delta_{S^{d-1}} g$ or $\Delta_{0} g$ for shortness. Hence for $x \in S^{d-1}$

$$
\Delta g(x)=k(k+d-2) g(x)+\Delta_{0} g(x), \quad x \in S^{d-1} .
$$

As we recall later this relation is very useful in the study of the spherical Laplacian. Combining (3.1) with the later relation and $x \cdot \nabla g=k g$, yields for $x \in S^{d-1}$,

$$
\frac{1}{2}\left(\Delta_{0} g(x)+k(k+d-2) g(x)\right)+k g(x)=\lambda g(x) .
$$


Therefore as a function on the sphere $g$ satisfies

$$
\Delta_{0} g=(2 \lambda-2 k-k(k+d-2)) g
$$

Since $g$ is homogeneous and non-zero on $\mathbb{R}^{d}$ it is non-zero on the sphere. Therefore $g$ is an eigenfunction of the spherical Laplacian. This operator is well studied: its eigenfunctions are the restrictions to the sphere of homogeneous harmonic polynomials (the spherical harmonics, see e.g. [7]). Its spectrum is

$$
\operatorname{Spect}\left(\Delta_{S^{d-1}}\right)=\{-\ell(\ell+d-2) ; \ell \in \mathbb{N}\}
$$

as one can deduce it from (3.2). Consequently the eigenvalues of $L$ are of the form $-\lambda$ with

$$
\lambda \in\{0,1\} \bigcup\left((0,+\infty) \cap\left\{k+\frac{1}{2}(k(k+d-2)-\ell(\ell+d-2)) ; \quad k, \ell \in \mathbb{N}, k \geq 2\right\}\right) .
$$

In particular $\lambda \in \mathbb{N} / 2$, and showing that $\lambda \neq 1 / 2$ will be enough to show that there is no eigenvalue between -1 and 0 . The following lemma shows that $\lambda$ cannot take the value $1 / 2$. It also shows that the eigenvalue -1 only appears for functions of degree $d=1$. The proof is complete.

Lemma 7. Let $d \geq 2$ be an integer. Then for all $k, \ell \in \mathbb{N}$, with $k \geq 2$

$$
k+\frac{1}{2}(k(k+d-2)-\ell(\ell+d-2)) \notin\left\{\frac{1}{2}, 1\right\} .
$$

Proof. First note that

$$
\begin{aligned}
2 k+k(k+d-2)-\ell(\ell+d-2) & =k^{2}+d k-\left(\ell^{2}+(d-2) \ell\right) \\
& =\left(k+\frac{d}{2}\right)^{2}-\left(\frac{d}{2}\right)^{2}-\left(\ell+\frac{d-2}{2}\right)^{2}+\left(\frac{d-2}{2}\right)^{2} \\
& =(k+\ell+d-1)(k-\ell+1)-(d-1) .
\end{aligned}
$$

Assume that $2 k+k(k+d-2)-\ell(\ell+d-2) \in\{1,2\}$. This is equivalent to

$$
(k+\ell+d-1)(k-\ell+1) \in\{d, d+1\} .
$$

By hypothesis $k+\ell+d-1 \geq d+1$. Hence the above inclusion forces $k-\ell+1=1$. Thus $k=\ell$ and $2 k+d-1 \in\{d, d+1\}$ or $2 k \in\{1,2\}$. This is a contradiction with $k \geq 2$.

Remark 1. The operator $L=\left(1-|x|^{2}\right) \Delta / 2-x \cdot \nabla, x \in B_{2}^{d}$ that we considered looks like the ultraspherical generators $\mathcal{L}_{m}$. Recall that for smooth enough $f$ on $]-1,1[$,

$$
\left.\mathcal{L}_{m} f(x)=\left(1-x^{2}\right) f^{\prime \prime}(x)-m x f^{\prime}(x), x \in\right]-1,1[.
$$

It has been studied in details, see e.g. [8]. Its spectral gap is of size $1 / m$. For integer values of $m, \mathcal{L}_{m}$ is the projection of the spherical Laplacian $\Delta_{S^{m}}$ onto ]-1, 1 . In dimension $d=1$, it holds $L=\mathcal{L}_{2} / 2$. Hence the knowledge of the spectral gap of $\mathcal{L}_{2}$ is another explanation for Theorem 5 in dimension $d=1$.

For $d \geq 2$, the operator $L$ does not coincide with the projection onto $B_{2}^{d}$ of the spherical Laplacian on $S^{d+1}$. However the reasoning of this note allows to study some properties of the spectrum of the $d$-dimensional analogues of $\mathcal{L}_{m}$, where the variable $x$ is in $B_{2}^{d}$.

Remark 2. It is not hard to check that on the equilateral triangle, linear functions do not have maximal variance among 1-Lipschitz functions. They are beaten by the distance to a vertex. 
Acknowledgements. We would like to thank Dominique Bakry and Naoufel Ben Abdallah for useful discussions.

\section{REFERENCES}

[1] M. Ajtai, J. Komlós and G. Tusnády, On optimal matchings. Combinatorica 4 (1984) 259-264.

[2] S.G. Bobkov and F. Götze, Exponential integrability and transportation cost related to logarithmic Sobolev inequalities. J. Funct. Anal. 163 (1999) 1-28.

[3] R.M. Dudley, The speed of mean Glivenko-Cantelli convergence. Ann. Math. Stat. 40 (1969) 40-50.

[4] A. Ganesh and N. O'Connell, Large and moderate deviations for matching problems and empirical discrepancies. Markov Process. Relat. Fields 13 (2007) 85-98.

[5] M. Ledoux, Sur les déviations modérées des sommes de variables aléatoires vectorielles indépendantes de même loi. Ann. Inst. H. Poincaré, Probab. Statist. 28 (1992) 267-280.

[6] M. Ledoux, Concentration of measure and logarithmic Sobolev inequalities, in Séminaire de Probabilités, XXXIII, Lect. Notes Math. 1709 120-216. Springer, Berlin (1999).

[7] C. Müller, Spherical harmonics IV. Springer-Verlag, Berlin-Heidelberg-New York (1966).

[8] C. Müller and F. Weissler, Hypercontractivity for the heat semigroup for ultraspherical polynomials and on the $n$-sphere. $J$. Funct. Anal. 48 (1982) 252-283.

[9] S.T. Rachev, Probability Metrics and the Stability of Stochastic Models. Wiley (1991).

[10] P.W. Shor, Random planar matching and bin packing, Ph.D. thesis, M.I.T., 1985.

[11] M. Talagrand, Matching theorems and empirical discrepancy computations using majorizing measures. J. Amer. Math. Soc. 7 (1994) 455-537.

[12] M. Talagrand, Transportation cost for Gaussian and other product measures. Geom. Funct. Anal. 6 (1996) 587-600.

[13] L. Wu, Large deviations, moderate deviations and LIL for empirical processes. Ann. Probab. 22 (1994) 17-27. 